\title{
CARACTERIZACIÓN FISICOQUÍMICA Y MICROBIOLÓGICA DE LA INFLUENCIA DE LOS RESIDUOS EN LA CALIDAD DEL AGUA DEL RÍO TOCANTINS, BANCO IZQUIERDO (MARAB-PA)
}

\section{ARTÍCULO ORIGINAL}

BITENCOURT, Emanoelen Bitencourt $\mathrm{e}^{1}$

ALMEIDA, Karina Miranda de ${ }^{2}$

SANTOS, Luana Mariza Morais dos ${ }^{3}$

JÚNIOR, Antônio Pereira ${ }^{4}$

BITENCOURT, Emanoelen Bitencourt e. Et al. Caracterización fisicoquímica y microbiológica de la influencia de los residuos en la calidad del agua del río Tocantins, izquierda Bank (MARAB-PA). Revista Científica Multidisciplinar Núcleo do Conhecimento. año 04, Ed. 06, Vol. 11, págs. 05-26. junio de 2019. ISSN: 24480959

\section{RESUMEN}

El agua es vital para la continuidad de la vida en el planeta, pero la calidad de los cuerpos de agua se ve alterada por la eliminación inadecuada de materiales orgánicos secos y húmedos, especialmente en el entorno urbano, incluso si hay parámetros y normas dirigidas a este problema. El objetivo de esta investigación fue la caracterización del agua del río Tocantins, en Marabá-PA, en relación con patrones de agua fisicoquímica y microbiológica, en dos puntos bajo la influencia de la

\footnotetext{
${ }^{1}$ Graduado en Ingeniería Ambiental.

${ }^{2}$ Graduado en Ingeniería Ambiental.

${ }^{3}$ Graduado en Ingeniería Ambiental.

${ }^{4}$ Máster en Ciencias Ambientales.
} 
eliminación de residuos, en la estación seca y lluviosa, y relacionar los datos obtenidos Con regulaciones legales. El método empleado fue el deductivo, el alcance de la investigación fue cuantitativo y cualitativo y el procedimiento fue experimental, realizado a partir del uso del Manual de funASA, SABESP Standard, método Winkler y SMWW, con la Asociación de Recopilación de Datos Documentos, cuyo recorte temporal fue entre 2010 y 2018. Los datos obtenidos indicaron que los parámetros físicos del agua están de acuerdo con lo establecido para aguas dulces Clase 2, Resolución ConAMA N. 357/2005, y en desacuerdo con los criterios de agua química. Los datos de las normas de baño indicaban agua inapropiada para la recreación del contacto primario, porque hay vertidos de restos residuales, que contienen sustancias como aceites y grasas, que pueden causar riesgos para la salud o comprometer la recreación, Según la resolución N. 274:2000. Por lo tanto, el tramo analizado del río está en disconformidad tanto en la clasificación de agua dulce como en la capacidad de baño.

Palabras clave: capacidad de baño, clasificación de agua dulce, parámetros de agua de calidad.

\section{INTRODUCCIÓN}

El agua es un recurso natural esencial para la vida en la Tierra. Por otro lado, la forma de utilizar este recurso, se produce varias veces, de forma inadecuada, lo que conduce a la no mantenimiento de la calidad, menor disponibilidad y alteración de las características naturales del afluente, que también cuenta con gas y solución sólida. Además de la contaminación natural, la gestión inadecuada de las zonas, la falta de tratamiento de aguas residuales y la eliminación indiscriminada de residuos son factores de gran importancia en la degradación de los recursos hídricos (BUZELLI; CUNHA-SANTINHO, 2013).

Además, los territorios urbanos y rurales, mediante la eliminación inadecuada de residuos y sustancias, contribuyen a los impactos en los sistemas hídricos. Ambos pueden colaborar con descargas de sustancias orgánicas e inorgánicas (algunas 
tóxicas), ya sea directamente en el cuerpo del agua, por filtración o infiltración de fuentes de contaminación, como productos utilizados para la producción y limpieza. Estos desplazamientos indebidos pueden causar daños a la fauna y enfermedades acuáticas de la población, además, el problema puede intensificarse por la degradación del medio ambiente, como la eliminación del bosque ribereño (BELLUTA et al., 2016; SCORSAFAVA et al., 2010).

En relación con los ríos urbanos, en los que las corrientes de agua tienen la ruta en las zonas urbanas, el cambio en la calidad del agua representa un riesgo para el progreso sostenible en estos lugares, especialmente en los países en desarrollo, como Brasil. Esto se debe a que la relación de los problemas de calidad en las masas de agua con la eliminación de residuos domésticos o incluso industriales en estos, todavía crudos, es decir, sin un tratamiento adecuado, provoca un aumento negativo de sustancias orgánicas e inorgánicas al río ( ISLAM et al., 2015; CHEN, 2017).

En este contexto, el problema de la eliminación inadecuada de materiales orgánicos secos y húmedos, y los impactos causados por éstos, en el medio ambiente, tiene como cuestión principal el destino adecuado de los restos de estos. Como resultado, se instituyó la Política Nacional de Residuos Sólidos (PNRS), Ley N. 12.305:2010, Decreto No. 7.404:2010, que tiene en el destino correcto de los residuos, con el fin de mitigar la cantidad producida por los generadores y los impactos ambientales. Por lo tanto, es necesario fortalecer los principios de gestión sostenible de residuos, por parte de los generadores y del municipio, para cumplir con las políticas establecidas (JACOBI; BESEN, 2011).

En consecuencia, en Brasil, la contaminación del agua es indudable, este hecho está asociado con la mala administración realizada en el país, incluso si hay recursos públicos invertidos para evitar esta situación crítica, además de la falta de rigor en el cumplimiento de las políticas de Aguas, necesitando un ciclo eficaz Plan, Hacer, Comprobar, Ley (PDCA, ${ }^{[5]}$ en inglés), para ayudar a las políticas públicas, a través de la determinación de acciones, ejecución, monitoreo y corrección del plan en su conjunto, para una mejor gestión, y la consiguiente conservación de la calidad de los 
recursos hídricos, especialmente en lo que respecta a la eliminación inadecuada de residuos (GONTIJO K-NIOR, 2013).

En cuanto a la legislación sobre la calidad del agua en Brasil, la resolución CONAMA N. 357 (BRAZIL, 2005), el agua del río Tocantins se clasifica como dulce Clase 2, en la que las aguas pueden ser destinadas:

(a) El suministro para el consumo humano, después del tratamiento convencional;

(b) La protección de las comunidades acuáticas;

c) La recreación del contacto primario, como natación, esquí acuático y buceo, de acuerdo con la Resolución N de CONAMA. 274 (BRASIL, 2000);

d) Riego de hortalizas, plantas frutales y parques, jardines, campos de deporte y ocio, con los que el público puede tener contacto directo; $Y$

(e) Actividades de acuicultura y pesca (BRASIL, 2005, p. 4).

En la misma resolución, en el arte. 15, las normas de agua dulce de la categoría 2 son las mismas que las aplicables al pedido 1, excepto:

II coliformes termotolerantes: Para el uso de la recreación de contacto primario debe obedecerse la resolución CONAMA N. 274 (BRASIL, 2000). Para otros usos, no se debe exceder un límite de 1.000 coliformes termotolerantes por cada 100 mililitros en un $80 \%$ o más de seis muestras recogidas durante un período de un año, con frecuencia bimensual. E. coli puede determinarse en sustitución de parámetros de coliformes termortolerantes de acuerdo con los límites establecidos por el organismo ambiental competente;

IV-turbidez: hasta 100 UNT; 
$\mathrm{V}-\mathrm{BOD} 5$ a $200 \mathrm{c}$ hasta $5 \mathrm{mg} / \mathrm{L} \circ 2$

VI-OD, en cualquier muestra, no menos de 5 mg/L O2 (BRAZIL, 2005, p. 10).

Otros patrones, por ejemplo, fósforo total (Ptotal) para el entorno óptico, nitrógeno total (Ntotal) y potencial hidromónico $(\mathrm{pH})$, también aparecen en esta resolución (tabla 1).

Tabla 1-límites de los estándares de calidad del agua dulce, Clase 2. CONAMA.

\begin{tabular}{|c|c|c|c|}
\hline Parâmetros & Mínimo & Máximo & Condição \\
\hline $\mathrm{DBO}_{5} 20^{\circ} \mathrm{C}$ & & $5 \mathrm{mg} / \mathrm{L}$ & \\
\hline Nitrato $\left(\mathrm{NO}^{-3}\right)$ & & $\begin{array}{l}10,0 \\
\mathrm{mg} / \mathrm{L}\end{array}$ & \\
\hline Nitrito $\left(\mathrm{NO}^{-2}\right)$ & & $\begin{array}{l}1,0 \\
\mathrm{mg} / \mathrm{L}\end{array}$ & \\
\hline \multirow[t]{4}{*}{ Nitrogênio amoniacal total } & & $\begin{array}{l}3,7 \\
\mathrm{mg} / \mathrm{L}\end{array}$ & $\mathrm{pH} \leq 7,5$ \\
\hline & & $2,0 \mathrm{mg} / \mathrm{L}$ & $\begin{array}{l}7,5<\mathrm{pH} \\
\leq 8,0\end{array}$ \\
\hline & & $\begin{array}{l}1,0 \\
\mathrm{mg} / \mathrm{L}\end{array}$ & $\begin{array}{l}8,0<\mathrm{pH} \\
\leq 8,5\end{array}$ \\
\hline & & $\begin{array}{l}0,5 \\
\mathrm{mg} / \mathrm{L}\end{array}$ & $\mathrm{pH}>8,5$ \\
\hline $\begin{array}{l}\text { Fósforo total (ambiente } \\
\text { lótico e tributários de } \\
\text { ambientes intermediários) }\end{array}$ & & $\begin{array}{l}0,1 \\
\mathrm{mg} / \mathrm{L}\end{array}$ & \\
\hline Oxigênio Dissolvido (OD) & $5 \mathrm{mg} / \mathrm{L}$ & & \\
\hline pH & 6,0 & 9,0 & \\
\hline Turbidez & & $\begin{array}{l}100 \\
\text { UNT }\end{array}$ & \\
\hline
\end{tabular}

Fuente: BRASIL (2005).

Subtítulos: DBO: Demanda bioquímica de oxígeno. pH.: Potencial hidromónico. NTU: unidades nefelométricas de turbidez

En el arte. 14, apartado I, de dicha resolución, se determina que los materiales sobrenadantes, como las espumas, aceites y grasas no naturales, las sustancias que 
interfieren con el sabor u olor, la coloración del origen antrópico y los residuos sólidos objetables deben Imperceptibles, es decir, no deben tener propiedades organolépticas.

Ya en la resolución N de CONAMA. 274 (BRAZIL, 2000), citado en el art. 15, párrafo II, Resolución N del CONAMA. 357 (BRAZIL, 2005), que se ocupa de los criterios de baño, clasifica el estado del agua dulce, salobre y agua salada en categorías propias e inadecuadas. En el arte. 2o, 1 de la resolución 274, las propias aguas se clasifican así a través de los resultados del conteo de coliformes, y se clasifican en:

A) Excelente: cuando en el $80 \%$ o más de un conjunto de muestras obtenidas en cada una de las cinco semanas anteriores, cosechadas en el mismo sitio, hay, como máximo, 250 coliformes fecales (termotolerantes) o 200 Escherichia coli o 25 Enterococcus para 100 mililitros;

b) Muy bueno: cuando en el $80 \%$ o más de un conjunto de muestras obtenidas en cada una de las cinco semanas anteriores, cosechadas en el mismo sitio, hay, como máximo, 500 coliformes fecales (termortolerantes) o 400 Escherichia coli o 50 Enterococcus para 100 mililitros;

c) Satisfactorio: Cuando en el $80 \%$ o más de un conjunto de muestras obtenidas en cada una de las cinco semanas anteriores, recogidas en el mismo sitio, hay un máximo de 1.000 coliformes fecales (termotolerantes) o 800 Escherichia coli o 100 Enterococcus para 100 mililitros ( BRASIL, 2000, pág. 257).

En cuanto a las inadecuadas, además de no cumplir con los estándares de sus propias aguas, se evalúan de otras maneras (n.o 4): 
b) El valor obtenido en el último muestreo es superior a 2500 coliformes fecales (termotolerantes) o 2000 Escherichia coli o 400 Enterococcus por 100 mililitros;

c) Incidencia alta o anormal en la región de enfermedades transmitidas por el agua indicadas por las autoridades sanitarias;

D) presencia de residuos o vertederos, sólidos o líquidos, incluidas aguas residuales sanitarias, aceites, grasas y otras sustancias, capaces de ofrecer riesgos para la salud o hacer que la recreación sea desagradable;

E) $6.0<\mathrm{pH}>9.0$ (Aguas Dulces), excepto por condiciones naturales; $</ \mathrm{pH}>$

f) Floración de algas u otros organismos, hasta que demuestre que no ofrecen riesgos para la salud humana;

g) Otros factores que contrarrestan, temporal o permanentemente, el ejercicio de la recreación de contacto primario (BRASIL, 2000, p. 257).

Así, la contaminación del agua por efluentes domésticos, debido a la deficiencia de saneamiento básico, es un hecho en los 143 municipios de Pará, por lo tanto, esta investigación está justificada, y estos hechos aumentan la relevancia de los mismos, cuyo objetivo es caracterizar el agua Del río Tocantins, Marabá-PA, con respecto a los parámetros físicos, químicos y microbiológicos del agua, en dos puntos bajo la influencia de la liberación de residuos, en la estación seca y lluviosa, y relacionar los datos obtenidos con las normas y normas legales. 


\section{MATERIAL Y MÉTODOS}

Los materiales utilizados para la medición de parámetros ambientales como la temperatura y el potencial hidrogenónico $(\mathrm{pH})$ fueron cedidos por la Universidad Estatal de Pará - UEPA (tabla 2).

Cuadro 2-instrumentos utilizados para medir las variables ambientales. Marabá - PA.

\begin{tabular}{|c|c|c|c|}
\hline Instrumentos & Marca & Modelo & Propósito \\
\hline Termómetro & Instrutherm & $\begin{array}{l}\text { Tipo de } \\
\text { skein }\end{array}$ & $\begin{array}{l}\text { Medición de la temperatura del } \\
\text { agua in situ }\end{array}$ \\
\hline Medidor de Ph & Producto OEM * & & $\begin{array}{l}\text { Medición del potencial } \\
\text { hidrosómiico in situ del agua }\end{array}$ \\
\hline Turbidimeter & MS Tecnopon & $\begin{array}{l}1000 P \\
\text { TB }\end{array}$ & $\begin{array}{l}\text { Medición de la turbidez del agua } \\
\text { ex situ }\end{array}$ \\
\hline Escala & $\begin{array}{l}\text { KnWaagen } \\
\text { Field }\end{array}$ & $320 / 3 \mathrm{KN}$ & $\begin{array}{l}\text { Pesar botellas y medios de } \\
\text { cultivo }\end{array}$ \\
\hline Autoclave vertical & Primatec & Cs & $\begin{array}{l}\text { Cultivo esterilizante Medios para } \\
\text { análisis microbiológico }\end{array}$ \\
\hline $\begin{array}{l}\text { Invernadero } \\
\text { bacteriológico }\end{array}$ & $\begin{array}{l}\text { Tecnología } \\
\text { Ethik }\end{array}$ & 403-3D & $\begin{array}{l}\text { Incubar los medios de cultivo con } \\
\text { las muestras para analizar } \\
\text { coliformes totales }\end{array}$ \\
\hline Bath-Maria & Hemoquímica & HM 1003 & $\begin{array}{l}\text { Incubar los medios de cultivo con } \\
\text { muestras para analizar } \\
\text { coliformes termotolerantes }\end{array}$ \\
\hline $\begin{array}{l}\text { Agitador magnético } \\
\text { con calefacción }\end{array}$ & Quimis & Q261-22 & $\begin{array}{l}\text { Evaporar el agua de las muestras } \\
\text { para el análisis total de residuos }\end{array}$ \\
\hline $\begin{array}{l}\text { Invernadero para } \\
\text { secar }\end{array}$ & Deleo & $\begin{array}{l}\mathrm{A} 2 \mathrm{C} \\
0208\end{array}$ & Secar el residuo total \\
\hline
\end{tabular}

* Fabricante de equipos originales. 
Fuente: Autores (2018).

El método empleado fue el deductivo, sintetizado por Matias-Pereira (2016), porque el crecimiento de la población se produjo en la ciudad analizada, así como el estancamiento de la extensión del saneamiento básico, dificulta la calidad de los cuerpos de agua. La investigación tuvo alcance cuantitativo y cualitativo, con naturaleza aplicada y procedimiento exploratorio, descrito por Sakamoto y Silveira (2014).

Este método se asoció con una encuesta documental con recorte temporal entre 2010 y 2018, para obtener datos secundarios más recientes en enlaces electrónicos de libre acceso, Scientific Eletronic Library Online (SciELO), comisión de Mejora del personal del nivel superior (CAPES), Ministerio de Medio Ambiente (MMA), Ministerio de la Ciudad, Ayuntamiento de Marabá y Secretaría Municipal de Vigilancia Sanitaria de Marabá.

\subsection{FISIOGRAFÍA DE LA MUNICIPALIDAD}

La investigación se llevó a cabo en la ciudad de Marabá, ubicada en el sureste de la mesoregión Deense (Figura 1), y representada con la ayuda de OSGeo (2016), con sedes identificadas en coordenadas geográficas 05 o 21 ' 54 "Latitud Sur y 04 o 07 ' 24" Longitud WGr. 
Figura 1-a) mapa político de la ubicación de Pará; b) Mapa físico de la ubicación de Marabá; c) Vista superior de la zona temática de esta investigación: frente al mar de Marabá-PA.
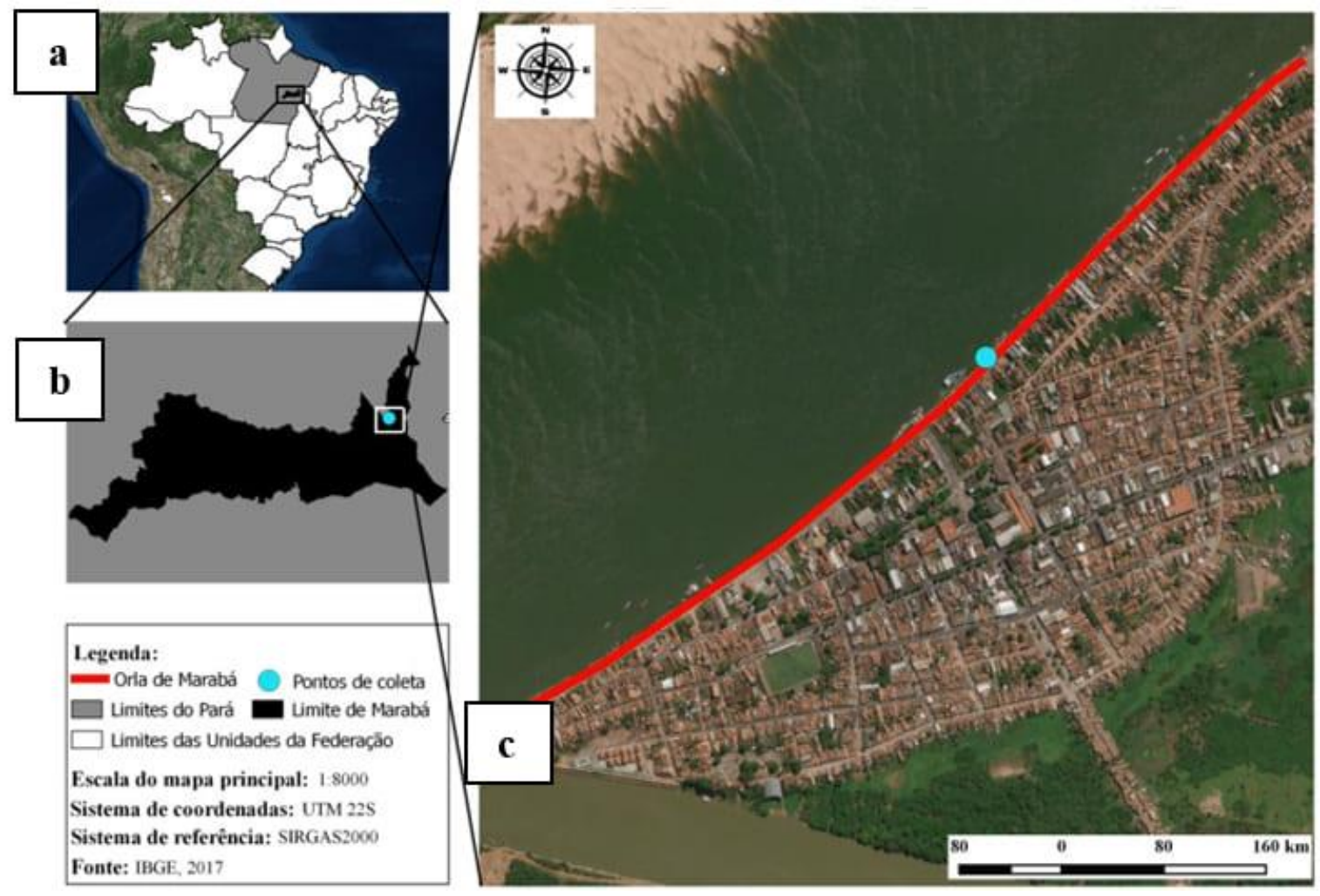

Fuente: Autores (2018).

En Marabá, los parámetros ambientales (precipitación, temperatura y humedad relativa del aire), presentan, anualmente, tendencias de variaciones por más o menos (tabla 3). 
Tabla 3-valores para promedios en las tres décadas climáticas analizadas. Marabá PA.

\begin{tabular}{|c|c|c|c|c|c|}
\hline \multicolumn{6}{|c|}{ Estação Marabá - OMM: 82562} \\
\hline $\begin{array}{l}\text { Décadas } \\
\text { analisad }\end{array}$ & $\begin{array}{l}\text { PPR total } \\
\text { (mm) }\end{array}$ & $\begin{array}{c}\text { Tmáxima } \\
\left({ }^{\circ} \mathrm{C}\right)\end{array}$ & $\begin{array}{c}\mathbf{T} \text { compensada } \\
\left({ }^{\circ} \mathrm{C}\right)\end{array}$ & $\mathbf{T}$ mínima $\left({ }^{\circ} \mathrm{C}\right)$ & UR $(\%)$ \\
\hline as & $\bar{X}$ & $\bar{X}$ & $\bar{X}$ & $\bar{X}$ & $\bar{X}$ \\
\hline $\begin{array}{c}1988- \\
1997\end{array}$ & 162,47 & 32,44 & 27,01 & 22,96 & 81,09 \\
\hline $\begin{array}{l}1998- \\
2007\end{array}$ & 144,97 & 33,14 & 27,67 & 23,69 & 73,87 \\
\hline $\begin{array}{l}2008- \\
2017\end{array}$ & 153,17 & 33,16 & 27,7 & 23,37 & 74,51 \\
\hline
\end{tabular}

Fuente: INMET (2018).

Subtítulos: PPR: Precipitación. T: Temperatura. RH: Humedad relativa del aire.

El intervalo de diciembre a mayo representa la estación lluviosa y, de junio a noviembre la estación seca, y la temperatura media anual es de 26,5 o C, (máximo 31,0 o C; mínimo 22,0 o C). El municipio tiene el índice Pluvimétrico de unos 2.000 $\mathrm{mm} / \mathrm{año}$, con alta humedad relativa (FAPESPA, 2016).

\subsection{MUESTREO}

El muestreo correspondió a ocho colecciones de agua, realizadas en el rango de diciembre de 2017 a septiembre de 2018 (cuatro en la temporada de lluvias; cuatro en seco), frente al caudal del río, según lo recomendado por la Fundación Nacional de la Salud (FUNASA, 2013) y la Guía Nacional Recogida y conservación de muestras (BRAND-O et al., 2011), en la margen izquierda del río Tocantins, Orla de MarabáPA, perímetro entre Av. Marechal Deodoro, Rua Santa Terezinha y Travessa Mestre Olivio, en dos puntos distintos: P1, Delatitude 5.346618 s y longitud 49.135026 o W; y P2, latitud 5.346257 s y longitud 49.134636 a W (Figura 2). 
Figura 2-Posiciones planialtimetricas de los puntos de recogida P1 y P2. Marabá - PA.

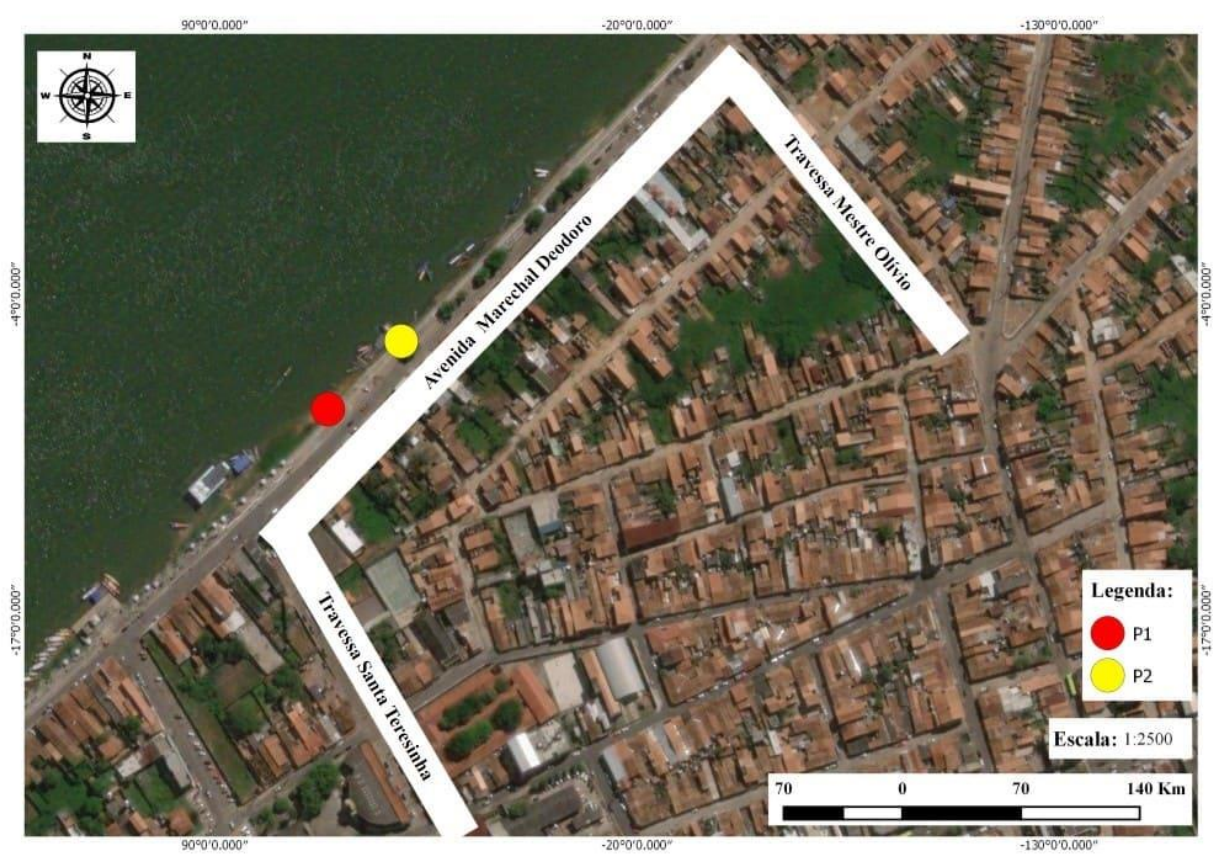

Fuente: Autores (2018).

Posteriormente, las muestras se realizaron para análisis de laboratorio, de acuerdo con lo recomendado por el Manual práctico de análisis de agua (FUNASA, 2013). Estos análisis se produjeron en el Laboratorio de Soluciones Agroindustriales VetPlus y se completaron en los laboratorios de clases prácticas (química; Ingeniería Ambiental y Forestal) del Campus VIII, Marabá - PA (tabla 4). 
Tabla 4-Especificaciones del sitio en el que se realizaron las pruebas de laboratorio, el tipo y el método empleados. Marabá - PA.

\begin{tabular}{|l|l|l|l|l|}
\hline Laboratorio & $\begin{array}{l}\text { Tipo } \\
\text { análisis }\end{array}$ & $\begin{array}{l}\text { Análisis de } \\
\text { laboratorio }\end{array}$ & $\begin{array}{l}\text { Método } \\
\text { utilizado }\end{array}$ & $\begin{array}{l}\text { Año de } \\
\text { publicación }\end{array}$ \\
\hline $\begin{array}{l}\text { Soluciones de } \\
\text { Agronegocios } \\
\text { VetPlus }\end{array}$ & Química & $\begin{array}{l}\mathrm{DB}^{\circ} 2 ; \quad \mathrm{N} \text { 3 } \\
\mathrm{PT}^{4}\end{array}$ & $\mathrm{SMWW}^{6}$ & 2012 \\
\hline $\begin{array}{l}\text { Química- UEPA } \\
\text { Ingeniería }\end{array}$ & Química & $\mathrm{OD}^{5}$ & $\begin{array}{l}\text { Winkler } \\
\text { modificado }\end{array}$ & S.D \\
\hline $\begin{array}{l}\text { Forestal } \\
\text { Ambiental - UEPA }\end{array}$ & físo & $\begin{array}{l}\text { Residuos } \\
\text { totales }\end{array}$ & $\begin{array}{l}\text { NTI 013 } \\
\text { SABESP7 }\end{array}$ & 1999 \\
\hline $\begin{array}{l}\text { Ingeniería } \\
\text { Ambiental - UEPA }\end{array}$ & Microbiológicos & Coliformes & FUNASA & 2013 \\
\hline
\end{tabular}

1Soluciones de Agronegocios; 2Demanda Bioquímica de Oxígen; 3Nitrógeno total 4Fósforo total; $50 \mathrm{x}$ disuelto; $6 \mathrm{Métod}^{\circ}$ s estándar para el examen del agua y las aguas residuales; 7No'ma Técnica Interna de la Empresa de Saneamiento Básico del estado de Sao Paulo.

Fuente: Autores (2018).

\subsection{TRATAMIENTO ESTADÍSTICO DE DATOS}

En cuanto al tratamiento estadístico de los datos obtenidos, se utilizaron estadísticas descriptivas, por la presencia de datos cuantitativos, continuos e intervalos, utilizando: A partir de la media, la desviación estándar (para observar la dispersión de las observaciones Los parámetros ambientales en relación con los valores intermedios) y el coeficiente de variación (para verificar la homogeneidad del conjunto de muestras obtenido), además de la correlación de Pearson, cuyas can tidades para r se adaptaron 
de las utilizadas por Porto, Jesús y Pereira Junior (2017), con patrones positivos y negativos (tabla 5).

Tabla 5-valores utilizados para $R$.

\begin{tabular}{|l|l|l|}
\hline Valores & Caracterización & Relación \\
\hline 0 & No hay & No hay \\
\hline 0.10 a 0.30 & Correlación débil & Directa \\
\hline-0.10 A-0.30 & Correlación débil & Marcha atrás \\
\hline 0,40 a 0,60 & Correlación media & Directa \\
\hline-0.40 A-0.60 & Correlación media & Marcha atrás \\
\hline 0.70 a 1.00 & Fuerte correlación & Directa \\
\hline-0.70 A-1.00 & Fuerte correlación & Marcha atrás \\
\hline
\end{tabular}

Fuente: Adaptado de los datos contenidos en Oporto, Jesús y Pereira Júnior (2017).

Utilizamos hojas de cálculo electrónicas contenidas en el software Excel (MICROSOFT CORPORATION, 2016) en el programa computacional BioEstat 5.3 (AYRES et al., 2007).

\section{RESULTADOS Y DISCUSIÓN}

\subsection{PARÁMETROS FÍSICOS DEL AGUA}

El análisis de los datos obtenidos para el pH indicó que, en ambos períodos, los valores obtenidos en P1 y P2 están comprendidos dentro de las normas establecidas $(6,0 \circ \mathrm{pH}$ a 9,0) en la resolución N. 357 (BRASIL, 2005). 
Tabla 5-Valores medios para pH (media, desviación estándar, coeficiente de variación) obtenidos para P1 y P2, estación lluviosa y seca. Marabá - PA.

\begin{tabular}{|c|c|c|c|c|}
\hline \multicolumn{5}{|c|}{$\mathbf{P}_{\mathbf{1}}$} \\
\hline & $\overline{\mathbf{X}}$ & & $\boldsymbol{\sigma}$ & $\mathbf{C V}(\mathbf{\%})$ \\
\hline $\mathbf{P C}$ & 7.39 & \pm & 0.10 & 1.35 \\
\hline $\mathbf{P S}$ & 7.23 & $\mathbf{\pm}$ & 0.28 & 3.87 \\
\hline \multicolumn{5}{|c|}{$\mathbf{P}_{\mathbf{2}}$} \\
\hline & $\overline{\mathbf{X}}$ & & $\boldsymbol{\sigma}$ & $\mathbf{C V}(\mathbf{\%})$ \\
\hline PC & 7.23 & \pm & 0.24 & 3.31 \\
\hline PS & 7.10 & \pm & 0.08 & 1.12 \\
\hline
\end{tabular}

Fuente: Autores (2018)

Subtítulos: pH.: Potencial hidromónico; PC: Temporada de lluvias. PD: Período seco;

: ${ }^{\bar{x}}$ Media: desviación estándar. CV (\%): Coeficiente de variación.

El análisis de los datos estadísticos permite afirmar que los valores obtenidos para el pH son bastante homogéneos, debido al promedio encontrado para la desviación estándar $(0,08,<\sigma>0,28)$ condensado alrededor de la media $(7,23) .</ \sigma>$

En el estudio realizado por Siqueira, Aprile y Miguéis (2012), en Parauapebas-PA, región sureste del estado, concluyeron que, para el $\mathrm{pH}$, existe una tendencia de elevación/disminución para los valores de este parámetro que se acercan a la neutralidad. Esto puede justificarse por la presencia de sedimentos e índices de alta materia orgánica presentes naturalmente en el sitio, y también indica que es común obtener valores bajos en la región amazónica. Los datos obtenidos en este estudio indican una tendencia a la neutralidad, con variaciones ligeramente ácidas, que corroboran el estudio en Parauapebas.

Aunque el río Tocantins tiene una fuente en el estado de Goiás, en la Serra Dourada, entre los municipios de Ouro Verde de Goiás y Petrolina de Goiás, tiene la desembocadura en Pará, y atraviesa el municipio de Marabá. En este caso, las 
características geológicas, como el predominio del suelo podzolico rojo-amarillo, y climática, como la temperatura media anual de los municipios (Marabá 26,5 o C; Los parauapebas $26,35 \circ \mathrm{C}$ ), son similares, lo que explica la similitud de los valores obtenidos.

Como se demostró en Marabá el lanzamiento de efluentes en los márgenes a partir de los cuales se realizaron las colecciones, la tendencia a la neutralidad puede justificarse por la oferta nutricional estable, es decir, las tasas de materia orgánica derivadas de los vertederos de efluentes a lo largo de la Los sedimentos suspendidos de origen natural no presentaron alteraciones importantes durante el período de investigación, lo que confiere un $\mathrm{pH}$ con un tendiente a la neutralidad, lo que revela similitud con la investigación realizada en Parauapebas.

Para la interrelación entre los parámetros del agua, los datos obtenidos indicaron que el $\mathrm{pH}$ está interferido con la temperatura del agua, especialmente durante la temporada de lluvias (P1, r a 0,43; P2, R -0,86), donde se vuelve ligeramente ácido. En la estación seca, la tendencia es a la alcalinidad $(P 1, r-0.14 ; \mathrm{P} 2, \mathrm{R}-0.65)$. En este caso, se verifica que estos parámetros son, en el cuerpo hídrico, cantidades inversamente proporcionales porque la temperatura, en el período seco, sube y la deposición de MO, llevada por la lluvia, en las disminuciones del cuerpo del agua, lo que puede Concentración del < od="'"> </>RE ( 9.38, $11.2 \mathrm{mg} / \mathrm{L})$, en P1 y P2, respectivamente (Figura 3). 
Figura 3 - Correlación entre la temperatura y el pH en el período seco en P1 y P2.

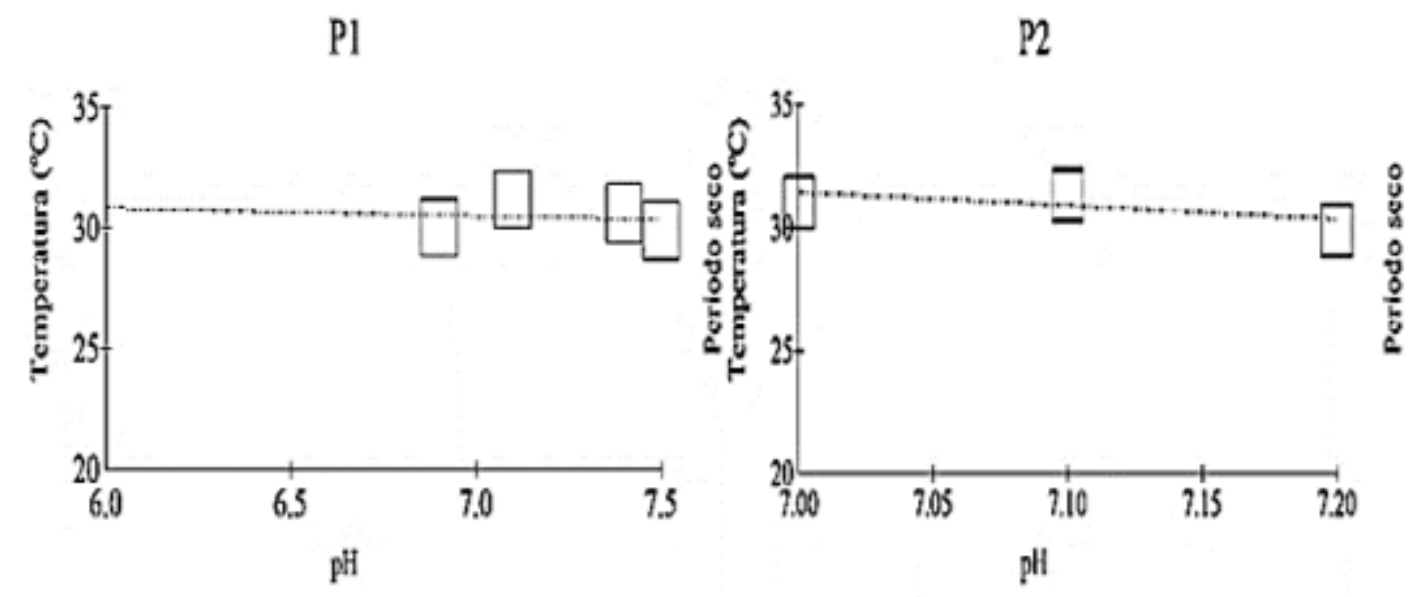

Fuente: Autores (2018)

En relación con los valores de temperatura medidos, no hubo diferencia significativa entre los puntos $\mathrm{P}_{1}$ e $\mathrm{P}_{2}$ en la estación lluviosa y en la estación seca (tabla 6).

Tabla 6-Valores medios para la temperatura (media, desviación estándar, coeficiente de variación) obtenidos para P1 y P2, estación lluviosa y seca. Marabá - PA.

\begin{tabular}{|c|c|c|c|c|c|}
\hline \multicolumn{5}{|c|}{$\mathrm{P}_{1}$} \\
\hline & $\overline{\mathbf{x}}$ & & $\boldsymbol{\sigma}$ & $\mathbf{C V}(\%)$ \\
\hline PC & 30.48 & \pm & 1.37 & 4.49 \\
\hline PS & 30.43 & \pm & 0.60 & 1.97 \\
\hline & \multicolumn{3}{|c|}{$\mathrm{P}_{2}$} \\
\hline & $\overline{\mathbf{x}}$ & & $\boldsymbol{\sigma}$ & $\mathbf{C V}(\%)$ \\
\hline PC & 30.43 & \pm & 1.40 & 4.60 \\
\hline PS & 30.9 & \pm & 0.69 & 2.23 \\
\hline
\end{tabular}

Fuente: Autores (2018)

Subtítulos: pH.: Potencial hidromónico. PC: Temporada de lluvias. PD: Período seco. N.o: Desviación estándar. CV (\%): Coeficiente de variación.

El análisis de los datos estadísticos permite afirmar que los valores obtenidos para la temperatura son ligeramente variados, debido a los promedios encontrados para la 
desviación estándar $(0,6<\sigma>, 1,4)$, con números más distribuidos alrededor de la media $(30,56) .</ \sigma\rangle$

La tendencia al equilibrio en los dos períodos analizados depende, internamente, de las variaciones estacionales y diurnas, además de la estratificación vertical. Externamente, latitud, altitud, estaciones, períodos del día, caudales y profundidad. Otro factor es el volcado de efluentes. En este caso, los puntos analizados reciben efluentes a través de arandelas. Sin embargo, este volcado no fue suficiente para causar elevación de la temperatura.

En la investigación realizada en llha do Marajó, por Alves et al. (2012), se indicó que los valores entre 27 oC y 31 oC pueden justificarse por las características naturales de las aguas amazónicas, donde las tendencias de variación de la temperatura se producen de acuerdo con las variaciones de los parámetros atmosféricos, por lo tanto, los Las temperaturas están relacionadas con el aumento de la cobertura de nubes, lo que disminuye la incidencia solar en el río, mientras que las más grandes cuando hay una mayor incidencia solar.

Estos cambios no se producen de un período estacional a otro, sino más bien en pequeñas variaciones a lo largo del año. Esto también se observó en este estudio, teniendo en cuenta que no hubo ninguna diferencia significativa en la temperatura en ambos períodos, lo que corrobora la investigación llevada a cabo en la isla de Marajó.

También se observa que los medios y la desviación estándar no presentaron discrepancias en los dos puntos, independientemente de las estaciones, lo que indica que las tendencias de elevación o disminución de la temperatura son independientes de los períodos estacionales, lo que corrobora con la investigación en la isla de Marajó.

Turbidez tiene como límite establecido hasta 100 unidades nefelométricas de Turbidez (UNT) por resolución N. 357 los valores obtenidos en este estudio variaron significativamente a lo largo de los períodos y según los puntos (tabla 7) (BRASIL, 2005). 
Tabla 7-Valores medios para turbidez (media, desviación estándar, coeficiente de variación) obtenidos para P1 y P2, estación lluviosa y seca. Marabá - PA.

\begin{tabular}{|c|c|c|c|c|}
\hline \multicolumn{5}{|c|}{$\mathrm{P}_{1}$} \\
\hline & $\overline{\mathbf{X}}$ & & $\boldsymbol{\sigma}$ & $\mathbf{C V}(\%)$ \\
\hline PC & 30.83 & \pm & 9.90 & 32.11 \\
\hline PS & 31.25 & \pm & 32.53 & 104.09 \\
\hline & \multicolumn{3}{|c|}{$\mathrm{P}_{2}$} \\
\hline & $\overline{\mathbf{X}}$ & & $\boldsymbol{\sigma}$ & $\mathbf{C V}(\%)$ \\
\hline PC & 24.85 & \pm & 12.50 & 50.30 \\
\hline PS & 37.6 & \pm & 38.60 & 102.65 \\
\hline
\end{tabular}

Fuente: Autores (2018).

Subtítulos: PC: Temporada de lluvias. PD: Período seco. ${ }^{\bar{x}}$ Promedio. N.o: Desviación estándar. CV (\%): Coeficiente de variación.

El análisis de los datos estadísticos permite afirmar que los valores obtenidos para la turbidez son bastante variados, así como la desviación estándar alta $(9,9,<\sigma>38,6)$, que está bien distribuida en torno a la media $(31,13) .</ \sigma\rangle$

Los datos obtenidos y analizados en los dos puntos de muestreo de este estudio, $e_{n}$ P1, la tendencia fue disminución (8 NTU), con distribución homogénea de las partículas (a 32,51 NTU; x a 31,25 NTU), y elevación en P2 (95 NTU). Esto puede estar relacionado con los períodos lluvioso y seco, lo que indica que existe una tendencia de baja dispersión de partículas que interfieren en la turbidez (por ejemplo, sólidos suspendidos, desechos orgánicos, etc.). En ambos puntos analizados, hay presencia de arandelas sanitarias de aguas residuales, sin embargo, su presencia no interfiere en la turbidez.

En el período pluvial, los datos analizados de P1 y P2 fueron bajos y más homogéneos, sin embargo en la sequía, estaban más dispersos, con un máximo en junio y agosto, respectivamente, todos están dentro de lo permitido. Aunque el promedio de los puntos no cambió drásticamente, el coeficiente de variación muestra que los datos 
tienen divergencias a lo largo de la investigación, lo que indica que los niveles de turbidez variaron no sólo a lo largo de los meses del año, sino también de una Otros, especialmente en los meses de sequía. Estos datos inesperados pueden ser explicados por Interferencias Antropas.

Buzelli y Cunha-Santino (2013), en un estudio realizado en Barra Bonita-SP, concluyeron que, aunque el índice de turbidez aumenta en el período lluvioso naturalmente debido a la carga de sedimentos en el agua, el vertedero de aguas residuales contribuye al aumento de partículas Suspensión y consecuente elevación de la turbidez en ambos periodos. En Marabá, el análisis de los datos obtenidos indicó que existe una similitud entre esta investigación y la realizada en Barra Bonita.

El análisis de los datos obtenidos del parámetro de residuo sin total indicó que había una tendencia a variaciones en las concentraciones de los residuos, que se calcularon únicamente para el valor intacto, sin fraccionamiento de los residuos (tabla 8).

Tabla 8 valores medios para residuos totales (media, desviación estándar, coeficiente de variación) obtenidos para P1 y P2, estación lluviosa y seca. Marabá - PA.

\begin{tabular}{|c|c|c|c|c|}
\hline \multicolumn{5}{|c|}{} \\
\hline & $\overline{\mathbf{X}}$ & & $\boldsymbol{\sigma}$ & $\mathbf{C V}(\%)$ \\
\hline PC & 325.00 & \pm & 378.59 & 116.48 \\
\hline PS & 325.00 & \pm & 206.16 & 63.43 \\
\hline & \multicolumn{3}{|c|}{$\mathrm{P}_{2}$} \\
\hline & $\overline{\mathbf{X}}$ & & $\boldsymbol{\sigma}$ & $\mathbf{C V}(\%)$ \\
\hline PC & 250.00 & \pm & 4767.60 & 186.96 \\
\hline PS & 250.00 & \pm & 238.05 & 95.22 \\
\hline
\end{tabular}

Fuente: Autores (2018).

Subtítulos: PC: Temporada de lluvias. PD: Período seco. ${ }^{\bar{x}}$ Promedio. N.o: Desviación estándar. CV (\%): Coeficiente de variación. 
El coeficiente de variación de los datos indica que difieren tanto en comparación con los períodos como en relación con los puntos. Sin embargo, los promedios permanecen en los puntos en los que se compara la temporada de lluvias con la seca.

En ambos puntos, los valores analizados pueden estar relacionados con la presencia de vertido de efluentes no tratados, residuos y actividades pesqueras en el sitio, como se ve en la investigación realizada por Silva y Araújo (2017), en Sao Paulo-SP, y que se observó en esta investigación Cerca de los sitios de recopilación, un factor determinante en los datos de este parámetro durante los períodos.

\subsection{PARÁMETROS QUÍMICOS DE AGUA}

Los datos obtenidos para bod indicaron que las concentraciones, tanto en P1 como en P2, en promedio, fueron más altas, en dos a cinco veces más en comparación con el valor de referencia permitido: $5 \mathrm{mg} / \mathrm{L}$ (tabla 9 ).

Tabla 9-Valores medios para DBO (media, desviación estándar, coeficiente de variación) obtenidos para P1 y P2, estación lluviosa y seca. Marabá - PA.

\begin{tabular}{|c|c|c|c|c|c|}
\hline \multicolumn{5}{|c|}{$\mathrm{P}_{1}$} \\
\hline & $\overline{\mathbf{X}}$ & & $\boldsymbol{\sigma}$ & $\mathbf{C V}(\%)$ \\
\hline PC & 5.90 & \pm & 4.43 & 75.08 \\
\hline PS & 4.95 & \pm & 3.10 & 62.62 \\
\hline & \multicolumn{3}{|c|}{$\mathrm{P}_{2}$} \\
\hline & $\overline{\mathbf{X}}$ & & $\boldsymbol{\sigma}$ & $\mathbf{C V}(\%)$ \\
\hline PC & 6.80 & \pm & 6.35 & 93.38 \\
\hline PS & 4.35 & \pm & 2.06 & 47.35 \\
\hline
\end{tabular}

Fuente: Autores (2018).

Subtítulos: DBO: Demanda bioquímica de oxígeno. PC: Temporada de lluvias. PD: Período seco. Promedio. N.o: Desviación estándar. CV (\%): Coeficiente de variación. 
El análisis de los datos obtenidos indicó mayores concentraciones de DBO, en P2, temporada de lluvias, en este caso, puede haber habido menos entrada de materia orgánica, por escorrente, o a través de la arandela de efluentes, ya que esto no tuvo agotamiento de la DBO, o incluso el La mineralización se produjo de forma lenta, ya que, en el tramo, hubo, en el mismo punto, una reducción (4,35 mg/L), por debajo del valor de referencia (BRAZIL, 2005).

Esta indicación se justifica por el hecho de que, durante la temporada de lluvias, la temperatura del agua tiende a equilibrarse, es decir, no sufre elevación, por lo que no hay pérdida expresiva de gas de oxígeno (O2) a la atmósfera, como en el período seco. Se observó que las concentraciones de DBO obtenidas en los meses de diciembre, enero y junio en ambos puntos están por encima del límite $(5 \mathrm{mg} / \mathrm{L})$ recomendado por la Resolución N. 357, así como la del mes de septiembre en p1 (BRAZIL, 2005).

En Rio Branco - AC, Santi et al. (2012) llevó a cabo una investigación sobre los valores de la DBO en los ríos, y los datos tratados están justificados por la alta presencia de aguas residuales sanitarias en la zona, y también se considera, desde el punto de vista geológico de la región amazónica, la presencia de materia orgánica originaria de Procesos naturales. Estos hechos también fueron verificados en Marabá-PA, ya que también se observó una gran concentración principalmente en la temporada de lluvias en p1 ya que está justificada por el valor de Pearson para la DBO y el fósforo total de 0.6787, que se caracteriza como una correlación directa ( Figura 4). 
Figura 4 - Correlación Pearson de DBO y fósforo total en la temporada de lluvias en P1.

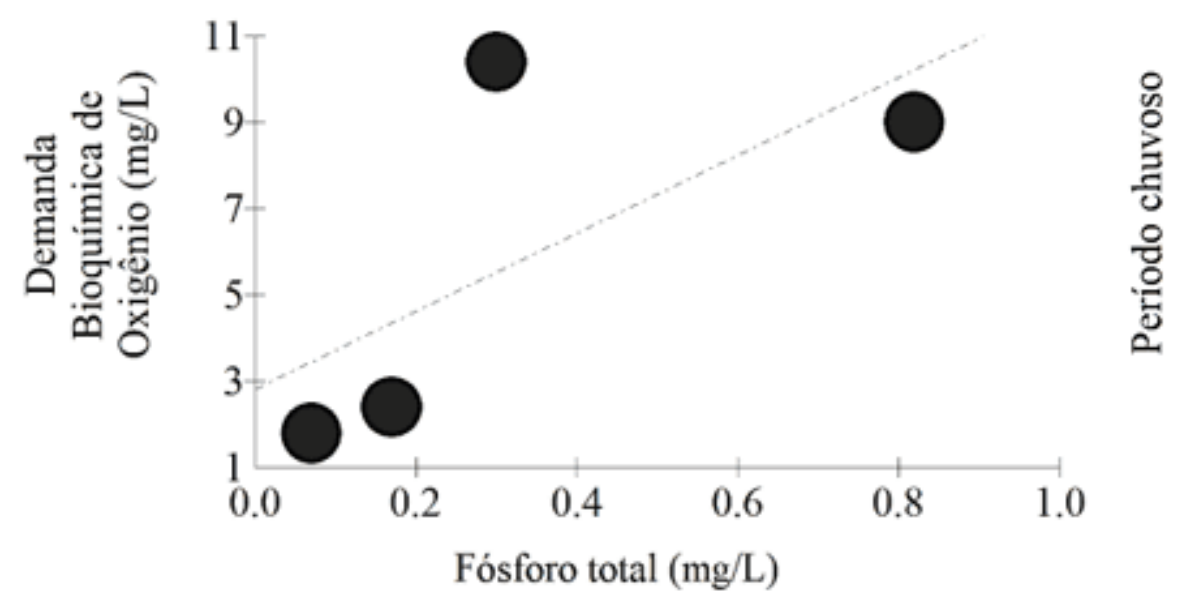

Fuente: Autores (2018).

En los meses de febrero, marzo, julio y agosto, en ambos puntos, y en el mes de septiembre $e_{n} \mathrm{P} 2$, las concentraciones de DBO se ajustan a las normas establecidas por la resolución N. 357, que puede referirse al caudal medio del río Tocantins antes de la estación seca y a la tendencia a aumentar la temperatura (BRAZIL, 2005).

En el período seco, los datos indicaron que tanto en $\mathrm{P}_{1}$ como en P2, había una tendencia a disminuir en las concentraciones de $\mathrm{DBO}<\mathrm{dbo}="$ "' $>(1,20,5 \mathrm{mg} / \mathrm{L})$. Estos valores están en no conformidad con la resolución recomendada CONAMA 357 (BRASIL, 2005), que establece un valor igual a $5 \mathrm{mg} / \mathrm{L}$.

Las tasas más bajas de DBO pueden estar relacionadas con la presencia de residuos generados en los establecimientos y residencias, en el agua, además de las actividades de pesca de peces en P1 (Figura 5a), así como el lanzamiento de aguas residuales no tratadas, que también se pueden En las proximidades de P2 (Figura 5b). 
Figura 5 - A) La presencia de actividades pesqueras cercanas a P1; B) Vertido de aguas residuales cerca del punto de recogida $\mathrm{P} 2$.

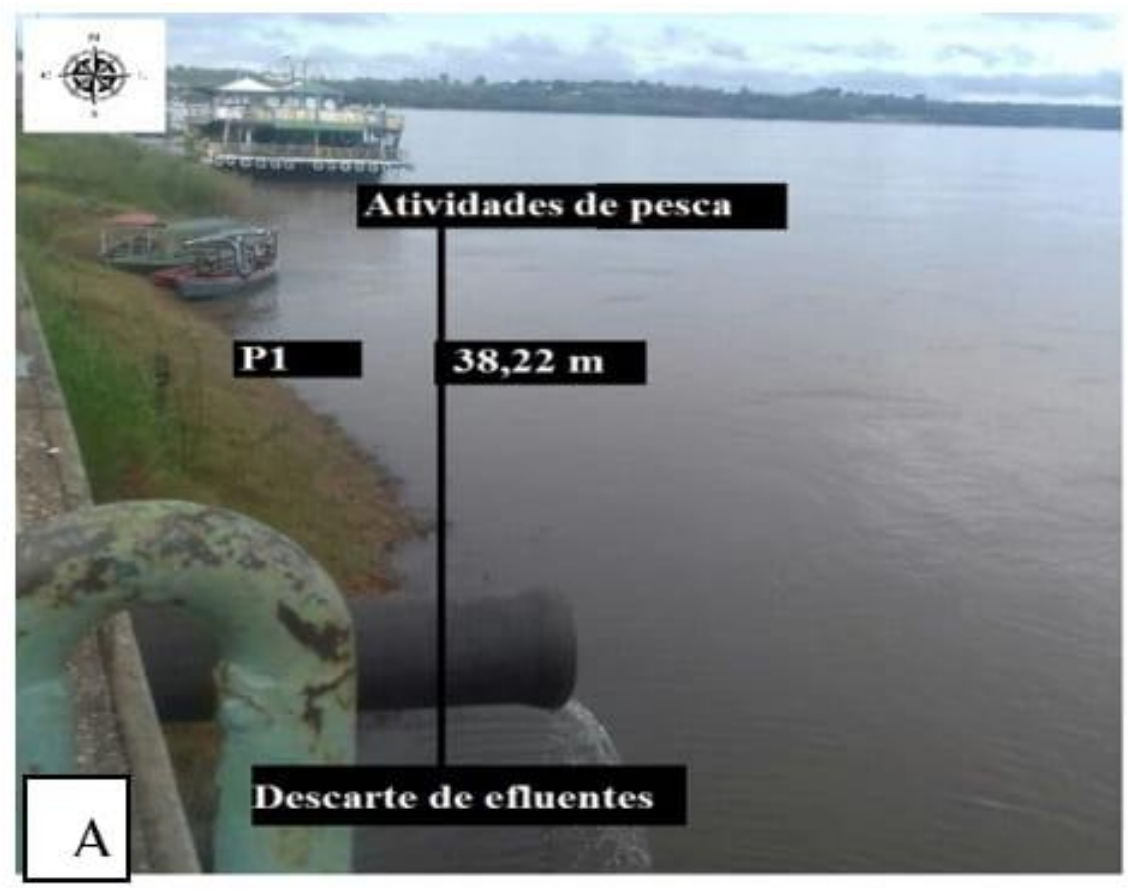

Fuente: Autores (2018)

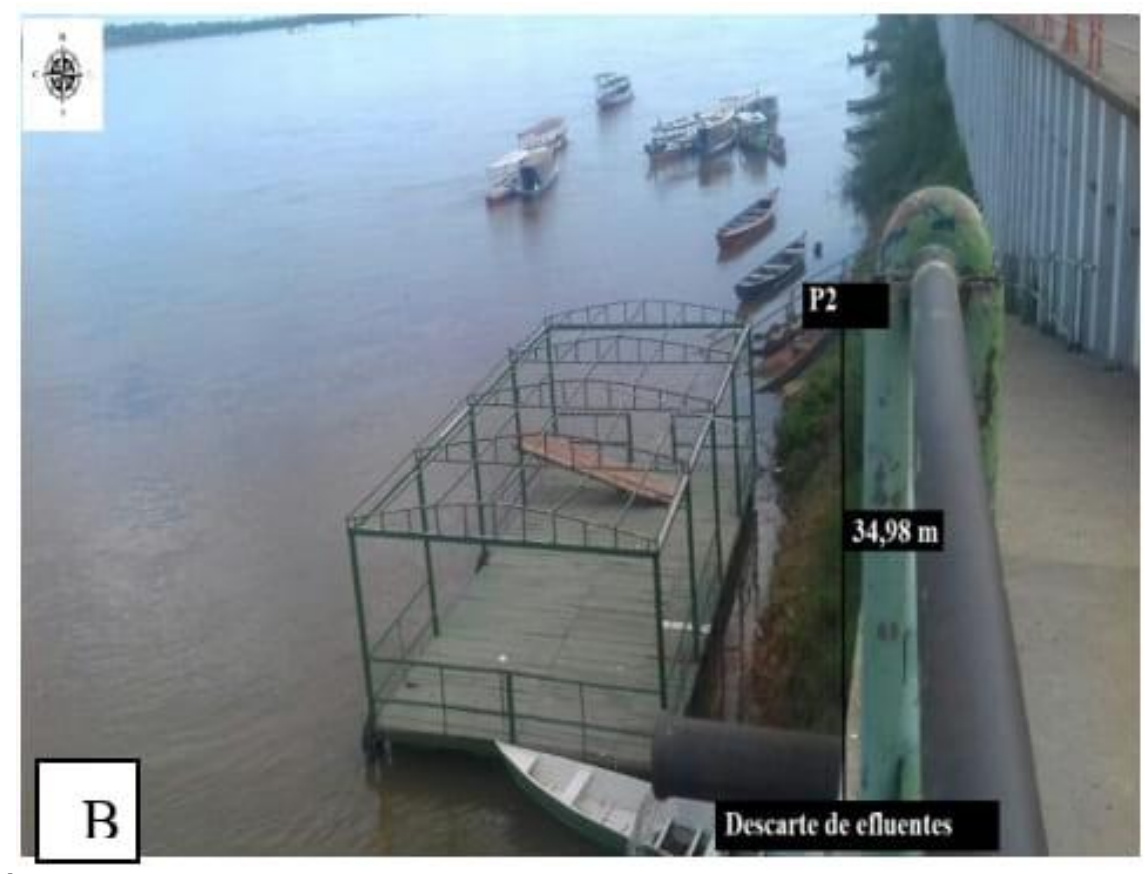

Fuente: Autores (2018).[/caption]

RC: 36695

Disponível em: https://www.nucleodoconhecimento.com.br/ingenieria-ambiental-es/influencia-de$\underline{\text { los-residuos }}$ 
En Braganza - PA, en el río Caeté (GORAYEB; Lombard PEREIRA, 2010), y en el río Araguari (B-B-B., 2010), este último pasa por tres municipios: Porto Grande, Ferreira y Cutias-PR, se realizaron estudios sobre las concentraciones de $\mathrm{DBO}$ en periodo seco. Los autores concluyeron que están interconectados la tendencia a aumentar la temperatura en el agua, así como la velocidad de flujo en el período anual. En el río Tocantins, los datos de estos parámetros pueden justificar las diferencias en las concentraciones debido a la influencia de estas variables, ya que las muestras se recogieron en dos períodos estacionales: lluviosos y secos.

En cuanto a las concentraciones de nitrógeno total, tanto en puntos como en período, los datos obtenidos indicaron sólo una concentración en desacuerdo con la recomendada $(3,7 \mathrm{mg} / \mathrm{L}$ para $\mathrm{pH}$ a 7,5$)$ en la resolución $\mathrm{N}$. 357, y estuvo en P1, en septiembre (cuadro 10) (BRASIL, 2005).

Tabla 10--valores medios para nitrógeno total (media, desviación estándar, coeficiente de variación) obtenidos para P1 y P2, estación lluviosa y seca. Marabá - PA.

\begin{tabular}{|c|c|c|c|c|}
\hline \multicolumn{5}{|c|}{$\mathrm{P}_{1}$} \\
\hline & $\overline{\mathbf{x}}$ & & $\boldsymbol{\sigma}$ & $\mathbf{C V}(\%)$ \\
\hline PC & 1.18 & \pm & 0.63 & 53.38 \\
\hline PS & 3.55 & \pm & 5.70 & 160.05 \\
\hline \multicolumn{4}{|c|}{$\mathrm{P}_{2}$} \\
\hline & $\overline{\mathbf{x}}$ & & $\boldsymbol{\sigma}$ & $\mathbf{C V}(\%)$ \\
\hline PC & 1.18 & \pm & 0.66 & 55.93 \\
\hline PS & 0.65 & \pm & 0.30 & 46.15 \\
\hline
\end{tabular}

Fuente: Autores (2018).

Subtítulos: NTotal: Nitrógeno total. PC: Temporada de lluvias. PD: Período seco. Promedio. N.o: Desviación estándar. CV (\%): Coeficiente de variación.

Estudio realizado por Santi et al. (2012), en Rio Branco - AC, concluyó que valores similares están justificados debido a los procesos de descomposición de la materia orgánica, liberando compuestos nitrogenados. Los datos obtenidos de las colecciones 
del río Tocantins, incluidas las normas anteriores, pueden estar relacionados con la presencia del punto de emisión de aguas residuales domésticas sin tratamiento previo cerca de los puntos, que se observa notablemente en la superficie del agua (Figura 6), en Desacuerdo con el arte. 14, párrafo I de la resolución CONAMA antes mencionada.

Figura 6 espumas del efluente volcado cerca de los puntos P1 y P2, Marabá-PA.

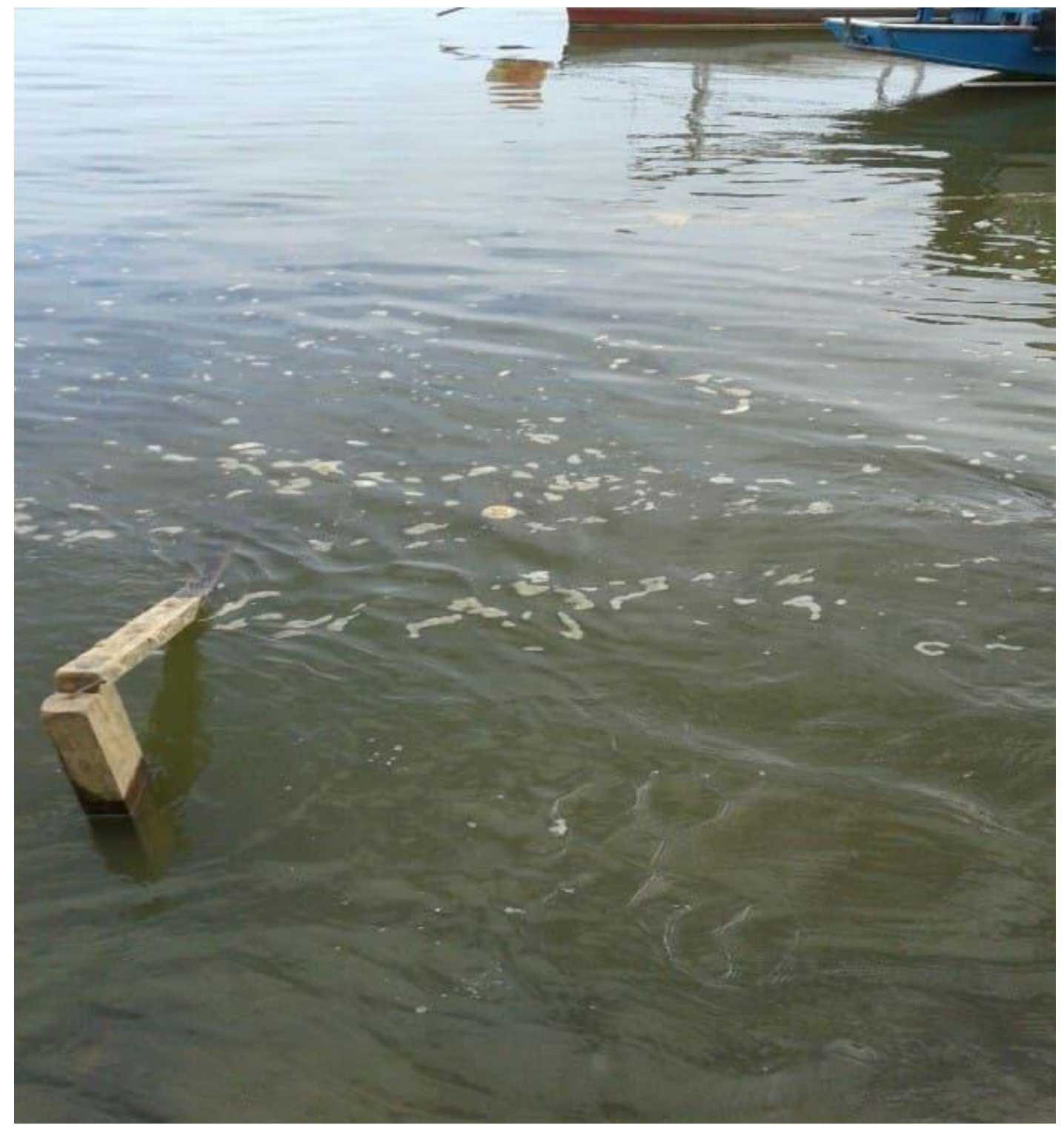

Fuente: Autores (2018) 
Como se muestra en la Figura 4, los valores obtenidos en la correlación de Pearson también destacan el exceso de carga orgánica en el río debido a la creciente proporción de nitrógeno y fósforo, que en la temporada de lluvias fueron 0.5988 en P1 y 0.7739 en P2, parámetros Directamente relacionado, mientras que en el período seco en los dos puntos de recogida, "R" fue mayor que 0,70 (P1 a 0,856; P2 a 0,8642), caracterizando la correlación entre estas variables como fuertes, directas y positivas (Figura 7).

Figura 7 - Gráficos de la correlación de Pearson de nitrógeno total y fósforo total de los puntos de recolección (P1 y P2) en la estación lluviosa y seca.

P1

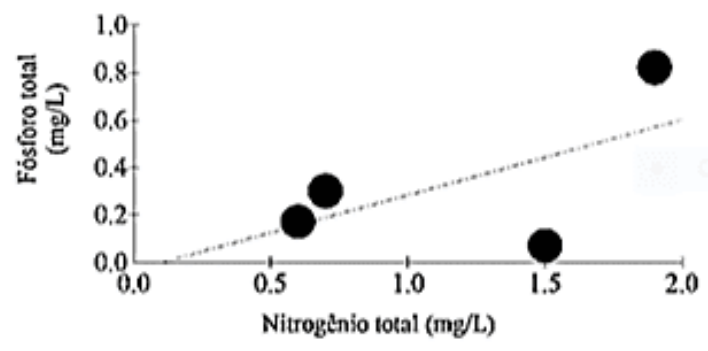

Pl

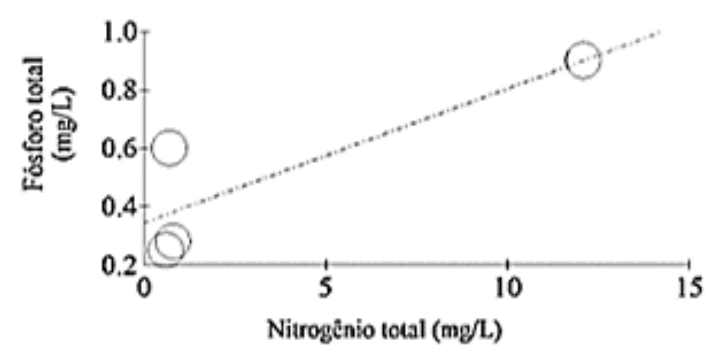

P2

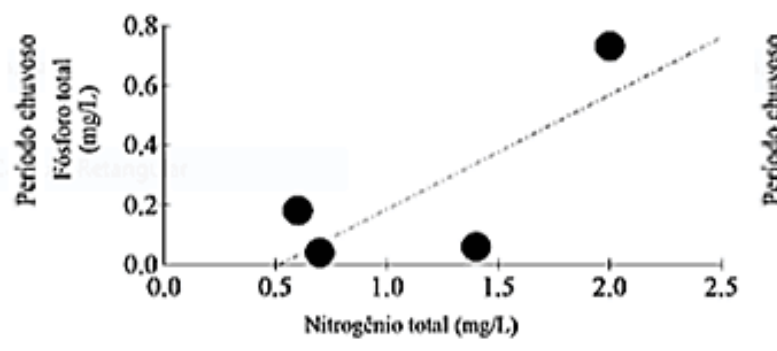

P2

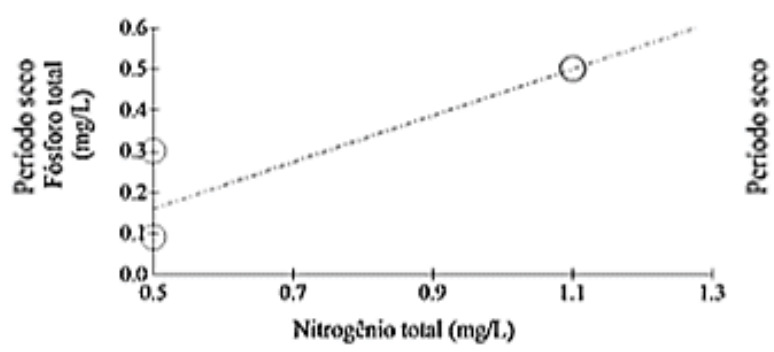

Fuente: Autores (2018)

Los valores permitidos para el fósforo total son equivalentes a $0,1 \mathrm{mg} / \mathrm{L}$, sin embargo, los datos de investigación en Marabá mostraron tendencias a la elevación en P1 y P2 (tabla 11). 
Tabla 11-Valores medios para fósforo total (media, desviación estándar, coeficiente de variación) obtenidos para P1 y P2, estación lluviosa y seca. Marabá - PA.

\begin{tabular}{|c|c|c|c|c|}
\hline \multicolumn{5}{|c|}{$\mathrm{P}_{1}$} \\
\hline & $\overline{\mathbf{X}}$ & & $\boldsymbol{\sigma}$ & $\mathbf{C V}(\%)$ \\
\hline PC & 0.34 & \pm & 0.33 & 97.05 \\
\hline PS & 0.51 & \pm & 0.31 & 60.78 \\
\hline \multicolumn{5}{|c|}{$\mathrm{P}_{2}$} \\
\hline & $\overline{\mathbf{X}}$ & & $\boldsymbol{\sigma}$ & $\mathbf{C V}(\%)$ \\
\hline PC & 0.25 & \pm & 0.32 & 128.00 \\
\hline PS & 0.13 & \pm & 0.11 & 84.61 \\
\hline
\end{tabular}

Fuente: Autores (2018)

Subtítulos: PTotal: Fósforo total. PC: Temporada de lluvias. PD: Período seco. Promedio. N.o: Desviación estándar. CV (\%): Coeficiente de variación.

La investigación realizada por Ríos-Villamizar, Martins Júnior y Waichman (2011) en los municipios de Boca do Acre y Beruri-AC, indicó una relación con valores similares y proximidad al principio y al final de la temporada de lluvias, donde la mayor carga detritica, erosiones y La carga de sedimentos se transporta en las aguas, además de la presencia de materia orgánica. Los valores máximos obtenidos en Marabá también se asemejan a estos periodos, corroborando la investigación realizada en Acre.

Los datos de oxígeno disuelto en la temporada de lluvias fueron elevados en P1 y P2, mientras que en la estación seca hubo una disminución (tabla 12). 
Tabla 12 valores medios para el oxígeno disuelto (media, desviación estándar, coeficiente de variación) obtenidos para $P_{1}$ y $P_{2}$, estación lluviosa y seca. Marabá PA.

\begin{tabular}{|c|c|c|c|c|}
\hline \multicolumn{5}{|c|}{$\mathrm{P}_{1}$} \\
\hline & $\overline{\mathbf{X}}$ & & $\boldsymbol{\sigma}$ & $\mathbf{C V}(\mathbf{\%})$ \\
\hline PC & 11.2 & $\mathbf{\pm}$ & 5.76 & 51.42 \\
\hline PS & 6.15 & \pm & 4.10 & 66.66 \\
\hline \multicolumn{5}{|c|}{$\mathrm{P}_{2}$} \\
\hline & $\overline{\mathbf{X}}$ & & $\boldsymbol{\sigma}$ & $\mathbf{C V}(\mathbf{\%})$ \\
\hline PC & 9.38 & \pm & 4.00 & 42.64 \\
\hline PS & 6.35 & \pm & 1.92 & 30.23 \\
\hline
\end{tabular}

Fuente: Autores (2018)

Subtítulos: OD: oxígeno disuelto. PC: Temporada de lluvias. PD: Período seco.

${ }^{\bar{x}}$ Promedio. N.o: Desviación estándar. CV (\%): Coeficiente de variación.

Los datos, en comparación con la resolución N de CONAMA. 357:2005, fue inferior a lo permitido ( $5 \mathrm{mg} / \mathrm{L}$ ), verificado en $\mathrm{P} 1$, en los meses de marzo, junio, julio y septiembre, ambos con una concentración de $4,1 \mathrm{mg} / \mathrm{L}$. En el estudio realizado por Oliveira E Cunha (2014), en Laranjal do Jari - AP, se llegó a la conclusión de que la alta temperatura disminuye la solubilidad del oxígeno en el agua debido a la alta incidencia solar.

\subsection{PARA ANÁLISIS MICROBIOLÓGICO}

El análisis de los datos obtenidos para coliformes totales indicó que, tan to en P1quanto en $\mathrm{P} 2$, se está produciendo, en ambos períodos de recolección, y esto es perjudicial tanto la calidad del agua como el baño del río Tocantins, ya que, frente al lugar donde se encuentra el Investigación, hay un alvius que la población de la zona llama "Playa Tucunaré", muy frecuada por los bañistas durante el verano amazónico. 
Los promedios del número más probable (NMP) del total de coliformes, tanto en las estaciones lluviosas como secas, en ambos puntos, superaron los 1.000/100 ml (tabla 13).

Tabla 13 valores medios para coliformes totales (media, desviación estándar, coeficiente de variación) obtenidos para P1 y P2, estación lluviosa y seca. Marabá PA

\begin{tabular}{|c|c|c|c|c|}
\hline \multicolumn{5}{|c|}{$\mathrm{P}_{1}$} \\
\hline & $\overline{\mathbf{X}}$ & & $\boldsymbol{\sigma}$ & $\mathbf{C V}(\%)$ \\
\hline PC & 1600 & \pm & 0 & 0 \\
\hline PS & 1600 & \pm & 0 & 0 \\
\hline & \multicolumn{3}{|c|}{$\mathrm{P}_{2}$} \\
\hline & $\overline{\mathbf{X}}$ & & $\boldsymbol{\sigma}$ & $\mathbf{C V}(\%)$ \\
\hline PC & 1425 & \pm & 350 & 24.56 \\
\hline PS & 1600 & \pm & 0 & 0 \\
\hline
\end{tabular}

Fuente: Autores (2018)

Subtítulos: ColTotal: Coliformes total. PC: Temporada de lluvias. PD: Período seco.

${ }^{\bar{x}}$ Promedio. N.o: Desviación estándar. CV (\%): Coeficiente de variación.

Los datos obtenidos indicaban un solo mes en el que el NMP era inferior a 1600 en marzo en p2. Estos altos valores pueden justificarse por el vertido directo de aguas residuales que se produce en la Orla de Marabá. En la investigación llevada a cabo por Martins et al. (2017), en los municipios de Joao Pessoa y Cabedelo - PB, en la playa de Cabo Branco, indicó la influencia de las aguas residuales en la calidad del agua, que se consideró inadecuada para la farnabilidad. Sin embargo, en el mes de agosto, en $\mathrm{P}_{2}$, la determinación del NMP se vio afectada, porque la combinación de tubos positivos obtenidos (5-0-5) no se encuentra en la tabla del Manual de FUNASA (2013). 
Los promedios del número más probable (NMP) de los coliformes termotolerantes, tanto en las estaciones lluviosas como secas, en ambos puntos, también superaron los $1.000 / 100 \mathrm{ml}$ (tabla 14).

Tabla 14 valores medios para coliformes termortolerantes (media, desviación estándar, coeficiente de variación) obtenidos para P1 y $P_{2}$, estación lluviosa y seca. Marabá - PA.

\begin{tabular}{|c|c|c|c|c|}
\hline \multicolumn{5}{|c|}{$\mathrm{P}_{1}$} \\
\hline & $\overline{\mathbf{X}}$ & & $\boldsymbol{\sigma}$ & $\mathbf{C V}(\%)$ \\
\hline PC & 1366.667 & \pm & 404.1452 & 29.5716 \\
\hline PS & 1600 & \pm & 0 & 0 \\
\hline & \multicolumn{4}{|c|}{$\mathrm{P}_{2}$} \\
\hline & $\overline{\mathbf{X}}$ & & $\boldsymbol{\sigma}$ & $\mathbf{C V}(\%)$ \\
\hline PC & 1425 & \pm & 350 & 24.5614 \\
\hline PS & 1287.5 & \pm & 625 & 48.54369 \\
\hline
\end{tabular}

Fuente: Autores (2018).

Subtítulos: ColTermo: Coliformes termotolerantes. PC: Temporada de lluvias. PD: Período seco. ${ }^{\bar{x}}$ Promedio. N.o: Desviación estándar. CV (\%): Coeficiente de variación.

Con respecto a este parámetro, los datos indicaron dos meses en los que el NMP fue inferior a 1600 en febrero, p1, y en marzo y julio, en p2. Además, el NMP fue indeterminado por las combinaciones de tubos positivos obtenidos en diciembre (4-54) y septiembre (0-5-3), en p1, ya que tampoco se muestran en la tabla del Manual de funASA (2013).

\section{CONCLUSIÓN}

La caracterización del agua del río Tocantins, Marabá - PA, en el período lluvioso y seco, en la sección analizada, que está influenciada por la liberación de residuos, indicada en cuanto a los parámetros hidrológicos físicos del agua, el cumplimiento de lo establecido para el agua dulce Clase 2, según la clasificación de la resolución $\mathrm{N}$ del 
CONAMA. 357 (BRASIL, 2005). Sin embargo, para la misma clase de agua, la caracterización de los parámetros hidrológicos químicos del agua indicó desacuerdo con esta resolución.

Para las normas de baño, según la resolución N. 274 (BRAZIL, 2000), el agua del río Tocantins se considera inapropiada para la recreación del contacto primario, ya que existe el vertido de sólidos y líquidos, que contienen sustancias como aceites y grasas que pueden causar riesgos para la salud o comprometer el ocio de la población.

\section{REFERENCIAS}

ALVES, I. C. C.et al. Qualidade das águas superficiais e avaliação do estado trófico do Rio Arari (Ilha de Marajó, norte do Brasil). Acta Amazônica, v. 42, n. 1, p.115-124, jan./dez. 2012.

AYRES, M. et al. BioEstat 5.3: aplicações estatísticas nas áreas das ciências biológicas e médicas. Belém: MCT; IDSM; CNPq, 2007. 364 p.

BÁRBARA, V. F. et al. Monitoramento sazonal da qualidade da água do rio Araguari/AP. Revista Biociências, Taubaté, v. 16, n. 1, p. 57-72, jul./dez. 2010.

BELLUTA, I. et al. Qualidade da Água, Carga Orgânica e de Nutrientes na Foz do Córrego da Cascata: Contribuição da Sub-Bacia para a Represa de Barra Bonita, Rio Tietê (SP). Revista Brasileira de Geografia Física, v. 09, n. 01, p. 305-318, jan./fev.2016.

BUZELLI, G. M.; CUNHA-SANTINO, M. B. Análise e diagnóstico da qualidade da água e estado trófico do reservatório de Barra Bonita, SP. Ambiente e Água - An Interdisciplinary Journal of Applied Science, Taubaté, v.8, n.1,p.186-205,abr.2013.

BRANDÃO, C. J. et al. (Org.). Guia Nacional de Coletas e Preservação de amostras: água, sedimentos, comunidades aquáticas e efluentes líquidas. Brasília: 
ANA-CETESB. Agência Nacional de Águas - Companhia de Saneamento do Estado de São Paulo, 2011.

BRASIL. Resolução n. 274, de 29 de novembro de 2000, do Conselho Nacional do Meio Ambiente - CONAMA. Revogada pela Resolução n. 357:2005. Define os critérios de balneabilidade em águas brasileiras. Diário Oficial [da] República Federativa do Brasil.n.18, de 25 de janeiro de 2001, Seção 1, páginas 70-71. Disponível em: <http://www.mma.gov.br/port/conama/legiabre.cfm?codlegi=272>. Acesso em: 06 fev. 2018.

BRASIL. Resolução n. 357, de 17 de março de 2005, do Conselho Nacional do Meio Ambiente - CONAMA. Alterada pela Resolução 410:2009 e pela 430:2011. Dispõe sobre a classificação dos corpos de água e diretrizes ambientais para o seu enquadramento, bem como estabelece as condições e padrões de lançamento de efluentes, e dá outras providências. Diário Oficial [da] República Federativa do Brasil. n. 053, de 18 de março de 2005, páginas. 58-63. Disponível em: <http://www.mma.gov.br/port/conama/res/res05/res35705.pdf>. Acesso em: 05 fev. 2018.

CHEN, W. Y. Environmental externalities of urban river pollution and restoration: a hedonic analysis in Guangzhou (China). Landscape and Urban Planning, v. 157, p. 170-179, 2017.

FAPESPA. FUNDAÇÃO AMAZÔNIA DE AMPARO A ESTUDOS E PESQUISAS. Estatísticas Municipais Paraenses: Marabá. Diretoria de Estatística e de Tecnologia e Gestão da Informação. Belém, n. 1, jul./dez. 2016. 60 f.

FUNASA.FUNDAÇÃO NACIONAL DE SAÚDE. Manual prático de análise de água. 4. ed. Brasília: Funasa, 2013. 150 p.

GONTIJO JÚNIOR, W. C. Uma avaliação da política brasileira de recursos hídricos baseada em dez casos de estudo. 2013. 330 f. Tese (Doutorado em Tecnologia Ambiental e Recursos Hídricos). Universidade de Brasília, Faculdade de 
Tecnologia, Programa de Pós-Graduação em Tecnologia ambiental e Recursos Hídricos. Brasília, 2013.

GORAYEB, A.; LOMBARDO, M. A.; PEREIRA, L. C. C. Qualidade da água e abastecimento na Amazônia: o exemplo da bacia hidrográfica do rio Caeté. Mercator - Revista de Geografia da UFC, Fortaleza. v. 9, n. 18, p. 135-157, jan./abr. 2010.

IBGE - Instituto Brasileiro de Geografia e Estatística. Mapas. Bases e Referenciais. 2017.

<ftp://geoftp.ibge.gov.br/organizacao_do_territorio/malhas_territoriais/malhas_munici pais/municipio_2015/Brasil/BR/>. Acesso em: 01 jun. 2018.

INMET -Instituto Nacional de Meteorologia. Banco de Dados Meteorológicos para Ensino Pesquisa. 2018Disponível em: $<$ http://www.inmet.gov.br/portal/index.php?r=bdmep/bdmep >. Acesso em: 25 fev. 2018.

ISLAM, M. S. et al. Heavy metal pollution in surface water and sediment: apreliminaryassessment of an urban river in a developing country. Ecological Indicators, v. 48, p. 282-291, 2015.

JACOBI, P. R.; BESEN, G. R. Gestão de resíduos sólidos em São Paulo: desafios da sustentabilidade. Estudos Avançados, São Paulo, v. 25, n. 71, p. 135-158, abr. 2011.

MARTINS, L. M. M. et al. Análise dos parâmetros de Balneabilidade: um estudo de caso sobre as praias dos municípios de João Pessoa e Cabedelo/PB. Revista InterScientia, João Pessoa, v. 5, n. 1, p. 116-128, abr. 2017.

MATIAS-PEREIRA, J. Manual de metodologia da pesquisa científica. 4. ed. São Paulo: Atlas, 2016.

MICROSOFT CORPORATION. Inc. Excel. Versão 1811. Software, 2016. 
OLIVEIRA, B. S. S.; CUNHA, A. C. Correlação entre qualidade da água e variabilidade da precipitação no sul do Estado do Amapá. Revista Ambiente e Água, Taubaté, v. 9, n. 2, p. 261-275, abr./jun.2014.

OSGEO - Open Source Geoespatial Foundation. Inc. QGis. Versão 2.18. Software, 2016.

PORTO, M. L.; JESUS, E. S.; PEREIRA JUNOR, A. Análise das tendências nas relações entre fluxo de veículos, arborização e os níveis de intensidade de ruído. Ecologia e Nutrição Florestal, Santa Maria, v.5, n.3, p.87-97, set./dez. 2017.

RÍOS-VILLAMIZAR, E. A.; MARTINS JÚNIOR, A. F.; WAICHMAN, A. V. Caracterização físico-química das águas e desmatamento na Bacia do rio Purus, Amazônia Brasileira Ocidental. Revista Geográfica Acadêmica, Goiânia, v.5, n.2, p. 54-65, jul./dez. 2011.

SAKAMOTO, C. K.; SILVEIRA, I. O. Como fazer projetos de iniciação científica. São Paulo: Paulus, 2014.

SANTI, G. M. et al. Variabilidade espacial de parâmetros e indicadores de qualidade da água na sub-bacia hidrográfica do igarapé São Francisco, Rio Branco, Acre, Brasil. Ecología Aplicada, Lima, v. 11 n. 1, p. 23-31, jan./ago. 2012.

SCORSAFAVA, M. A. et al. Avaliação físico-química da qualidade de água de poços e minas destinada ao consumo humano. Revista do Instituto Adolfo Lutz, São Paulo, v. 69, n. 2, p. 229-232, abr./jun. 2010.

SILVA, M. A.; ARAÚJO, R. R. Análise temporal da qualidade da água no córrego limoeiro e no Rio Pirapozinho no estado de São Paulo - Brasil. Revista FORMAÇÃo (ONLINE), São Paulo, v. 1, n.24, p. 182-203, jan. /abr. 2017.

SIQUEIRA, G. W.; APRILE, F.; MIGUÉIS, A. M. Diagnóstico da qualidade da água do rio Parauapebas (Pará - Brasil). Acta Amazonica, Manaus, v. 42, n. 3, p. 413-422, jul. /set. 2012. 
5.Planejar, agir, checar e corrigir.

Enviado: Febrero, 2019.

Aprobado: Junio de 2019. 